\title{
Avaliação clínica de palidez estratificada em cruzes: concordância entre observadores e comparação com níveis séricos de hemoglobina
}

\author{
Clinical evaluation of cross-stratified pallor: agreement between observers and \\ comparison with the hemoglobin levels
}

Rosemeri Maurici da Silva, Laura Bittencourt, Thiago Mamôru Sakae

\section{Resumo}

Objetivo: comparar o grau de palidez, estratificada em cruzes, com os níveis laboratoriais de hemoglobina e avaliar o grau de concordância entre observadores. Casuística e métodos: foram avaliados, no período de março a abril de 2007, por meio de estudo transversal observacional, os indivíduos com idade superior a 18 anos admitidos em cinco unidades do Hospital Nossa Senhora da Conceição, em Tubarão, Santa Catarina, para os quais haviam sido solicitadas dosagens de hemoglobina. Os pacientes foram avaliados por dois observadores quanto à presença e grau de palidez (de ausente $a++++/ 4$ ) nos seguintes locais: mucosa ocular, leito ungueal e cristas palmares. 0 índice de concordância Kappa foi utilizado avaliar a concordância entre observadores e o teste de KruskalWallis para avaliar a diferença entre a média dos níveis de hemoglobina para os diferentes graus de palidez atribuídos. Resultados: estudou-se uma amostra de 30 pacientes, com idade média de 63,1 anos, sendo 70\% do gênero masculino. A comparação entre os níveis médios de hemoglobina com a palidez graduada em cruzes não apresentou valores estatisticamente significativos para os diferentes graus atribuídos. 0 índice de concordância Kappa foi de 0,25 nas cristas palmares, 0,06 no leito ungueal e 0,16 na mucosa ocular. Conclusões: não houve boa concordância entre os observadores na avaliação de palidez estratificada em cruzes. Os níveis médios de hemoglobina não apresentaram diferenças estatisticamente significativas entre os diferentes graus de palidez atribuídos.

Palavras-Chave: Palidez; hemoglobina; anemia.

\section{Abstract}

Objective: to compare the degree of cross-stratified pallor with the hemoglobin levels and to evaluate the agreement degree between observers. Subjects and methods: in March-April 2007, an observational cross sectional study was carried out with individuals aged over 18 years admitted in five hospital units Hospital Nossa Senhora da Conceição, in Tubarão, Santa Catarina, for whom the dosages of hemoglobin had been requested. The patients were evaluated by two observers for the presence and degree of pallor (from absence to $++++/ 4$ ) in the following sites: ocular mucosa, ungual bed and palmar crests. The Kappa agreement index was used to evaluate the agreement between observers and the Kruskal-Wallis test was used to evaluate the difference between the mean hemoglobin levels for different degrees of observed pallor. Results: a sample of 30 patients, mean age 63.1 years, $70 \%$ male was analyzed. The most frequent response was $+/ 4$ in ungual bed and ocular mucosa, and absent in palmar crests. The comparison between the mean levels of hemoglobin with the cross-stratified pallor showed no statistically significant values for the different degrees assigned. The Kappa agreement index was 0.25 in palmar crests, 0.06 in ungual beds and 0.16 in the ocular mucosa. Conclusions: there was low agreement between the observers in evaluating cross-stratified pallor. Mean levels of hemoglobin showed no statistically significant differences between the various attributed degrees of pallor.

Keywords: Pallor; hemoglobin; anemia.

Recebido: 23/08/2007

Revisado: $10 / 03 / 2008$

Aprovado: 27/06/2008

Universidade do Sul de Santa Catarina (Unisul) e Hospital Nossa Senhora da Conceição, Tubarão, Santa Catarina, Brasil.

Endereço para correspondência: Rosemeri Maurici da Silva, Rua Moçambique, 852 - Rio Vermelho, CEP 88060-415 - Florianópolis (SC),

Tel/Fax: (48) 32697008, E-mail: rosemaurici@gmail.com 


\section{Introdução}

Denomina-se anemia a concomitância de síndrome clínica e quadro laboratorial decorrentes da baixa quantidade de eritrócitos circulantes no sangue. Este número reduzido ocorre como conseqüência do comprometimento na produção, aumento da destruição ou perda de eritrócitos, sendo manifestação comum de inúmeros distúrbios ${ }^{1-4}$.

A anemia afeta o desenvolvimento físico e cognitivo das crianças, a produtividade dos adultos e, possivelmente, aumenta o risco de morbimortalidade pós-operatórias ${ }^{1-6}$, gerando assim, sérias consequiências para a qualidade de vida dos indivíduos.

Cálculos recentes da Organização Mundial da Saúde (OMS) estimam a prevalência mundial de anemia em aproximadamente dois bilhões de indivíduos e que, possivelmente, metade das crianças e gestantes nos países em desenvolvimento apresente diagnóstico de anemia em algum momento de suas vidas 5 . Além disso, pode-se detectar algum grau de anemia em 20 a $40 \%$ dos pacientes hospitalizados ${ }^{2}$. No que se refere ao Brasil, e mais especificamente ao estado de Santa Catarina, foi realizado um estudo que detectou anemia em $40 \%$ das crianças, porcentagem esta que se torna ainda maior quando relativa às crianças de baixa renda? .

Na obtenção do diagnóstico, é importante considerar os dados de anamnese, exame físico e laboratoriais ${ }^{2,3}$. As principais manifestações clínicas consistem em sintomas variados, como palpitações, astenia, fadiga, dentre outros, que se associam conforme o tipo e etiologia da anemia. Ao exame físico, a palidez funciona como indicador $^{8}$, devendo ser avaliada em locais como cristas palmares, leitos ungueais e mucosas, uma vez que nestas áreas, os vasos encontramse próximos à superfície ${ }^{3}$. Essa manifestação semiológica da anemia pode ser verificada pela inspeção, sob a forma de descoramento ou palidez cutâneo-mucosa. Quando presente, o descoramento deve ser quantificado em escala de uma a quatro cruzes $(+/ 4,++/ 4,+++/ 4$ e $++++/ 4)$, sendo que o número de cruzes aumenta proporcionalmente à palidez ${ }^{8}$. É importante ressaltar, no entanto, que a anemia só é identificada à inspeção quando o nível de hemoglobina cai a valores inferiores a $8-10 \mathrm{~g} / \mathrm{dL}^{3}$.

Além dos parâmetros semiológicos, a anemia também deve ser avaliada através de análise laboratorial, estando presente quando os exames refletirem níveis de hematócrito $(\mathrm{Ht})$ e hemoglobina $(\mathrm{Hb})$ abaixo do esperado para indivíduos sadios de mesma idade e sexo. Os valores de hematócrito considerados normais em indivíduos adultos são 37 a $47 \%$ em mulheres e 40 a $54 \%$ em homens. Já a faixa normal de hemoglobina para mulheres e homens é de 12 a $16 \mathrm{~g} / \mathrm{dL}$ e 13 a $18 \mathrm{~g} / \mathrm{dL}$, respectivamente ${ }^{2,3,9}$.

De acordo com a OMS, a utilização do eritrograma com avaliação dos níveis de hematócrito e hemoglobina constitui uma boa escolha em saúde pública, pois é um exame simples e de baixo custo. Porém, em regiões sem disponibilidade de recursos, como países subdesenvolvidos ou em desenvolvimento, nem sempre sua realização é viável ${ }^{10}$. No Bra- sil, a dosagem de hemoglobina geralmente não é realizada sem que haja alterações na coloração detectada ao exame físico, ressaltando, portanto, a importância em se conhecer a precisão da avaliação da coloração cutâneo-mucosa no diagnóstico ${ }^{11}$.

Desse modo, se faz necessário conhecer a validade da semiologia quando comparada aos exames laboratoriais, com o intuito de oferecer aos pacientes o tratamento precoce e diminuir a quantidade de resultados falso-negativos e os riscos por eles oferecidos.

\section{Casuística e métodos}

Foram avaliados, no período de nove de março a 23 de abril de 2007, por meio de estudo transversal observacional, todos os indivíduos maiores de 18 anos, admitidos em quatro Unidades de Internação Clínica e na Unidade de Terapia Intensiva (UTI) do Hospital Nossa Senhora da Conceição (HNSC), em Tubarão, Santa Catarina, para os quais haviam sido solicitadas dosagens de hemoglobina no momento da admissão.

Os participantes ou seus responsáveis foram informados a respeito do estudo e concordaram em participar através de assinatura de termo de consentimento livre e esclarecido.

Realizado este procedimento, os participantes do estudo foram cadastrados em ficha de inclusão e avaliados em um período máximo de 24 horas após a admissão, à luz artificial.

Os avaliadores, por sua vez, após esclarecimento dos procedimentos a serem adotados, e sem conhecimento dos níveis laboratoriais $\mathrm{Hb}$, anotaram seu parecer em uma ficha fornecida pelos pesquisadores, após assinatura de termo de consentimento livre e esclarecido para participar da pesquisa. Os dados questionados foram: presença ou ausência de palidez; havendo palidez, a quantificação em cruzes (+/4 a $++++/ 4)$; e local do exame (mucosa ocular, cristas palmares, ou leito ungueal). Cada participante foi avaliado por dois profissionais, sem que tivessem conhecimento da análise do colega.

Foram excluídos do estudo, os indivíduos que não concordaram em participar através da assinatura do termo de consentimento livre e esclarecido. Excluíram-se, ainda, os pacientes que não puderam ser avaliados no máximo em 24 horas após a admissão e aqueles transfundidos durante este período.

Para a dosagem laboratorial de $\mathrm{Hb}$ foi utilizada a técnica da cianometahemoglobina, em equipamento $\mathrm{ABX} 60^{\circledR}$

Os dados da pesquisa foram armazenados em um banco de dados confeccionado através do software Epidata 3.1, e a análise estatística foi realizada com o software Epi-Info $6.04^{12}$.

O grau de concordância entre observadores foi estimado através do índice de concordância Kappa, sendo considerados satisfatórios valores maiores ou iguais a 0,75 . O teste de Kruskal-Wallis foi utilizado para avaliar as diferenças entre os níveis médios de hemoglobina para os diferentes graus de palidez atribuídos, sendo aceitos como significativos valores de $\mathrm{p}<0,05^{12}$ 
O projeto de pesquisa foi submetido e aprovado pelo Comitê de Ética e Pesquisa da Universidade do Sul de Santa Catarina (Unisul) sob o número 06.614.4.01.III.

\section{Resultados}

Foram avaliados, consecutivamente, 30 pacientes, dos quais $93,3 \%$ eram caucasianos. Em relação ao gênero, houve predomínio do masculino (70\%) sobre o feminino (30\%). A média de idade foi de 60,13 anos (desvio padrão (dp) 12,8), variando entre 38 e 83 anos.

Quanto aos valores de $\mathrm{Hb}$, a média total foi de $11,27 \mathrm{~g} / \mathrm{dL}$ $(\mathrm{dp}=2,48)$.

Cada participante foi avaliado por dois observadores que constituíram o grupo de observadores 1 e 2 .

A distribuição do grau de classificação de palidez em cruzes para os diferentes locais de avaliação, de acordo com a hemoglobina, encontra-se demonstrada nas Tabelas de 1 a 3 . Nenhum observador atribuiu à estratificação de palidez $++++/ 4$. Não foi observada diferença estatisticamente significativa entre os níveis médios de $\mathrm{Hb}$ e a graduação em cruzes atribuída em todos os locais examinados ( $p>0,05)$.

O índice de concordância Kappa foi de 0,25 nas cristas palmares, 0,06 no leito ungueal e 0,16 na mucosa ocular.

\section{Discussão}

Não existem dados, na literatura utilizada como referência, que atribuam maior dificuldade de observação de palidez em homens ou mulheres. No presente estudo, a proporção de diagnósticos concordantes ou discordantes foi semelhante entre os indivíduos do gênero masculino e feminino.

As condições ideais para examinar a coloração da pele é: iluminação natural sem incidência direta sobre a pele, no período diurno ${ }^{8}$. O presente estudo buscou avaliar os pacientes diante das condições reais oferecidas aos profissionais da área médica no serviço em que a pesquisa foi realizada.

Não há homogeneidade, segundo os diversos autores, quanto aos locais do corpo a ser pesquisada a palidez, tampouco, qual o melhor local para investigação $0^{11,13-17}$. Estudos anteriores mostraram diferentes resultados conforme o local avaliado ${ }^{13-17}$, fato não observado no presente estudo. Uma metanálise de estudos, realizados em crianças, encontrou melhores resultados nos sítios leito ungueal ou palmas das mãos, conforme o nível de hemoglobina ${ }^{18}$.

Quando comparados os níveis de hemoglobina com a graduação em cruzes para cada local, não houve diferença estatisticamente significativa em nenhum dos três sítios examinados ( $p>0,05)$. Kalter et al., em estudo africano aplicado em crianças, apesar de encontrarem baixa sensibilidade relativa ao descoramento palmar e conjuntival na avaliação da anemia severa, verificaram correlação entre o achado de palidez e os níveis de hematócrito e hemoglobina ${ }^{15}$.
O índice de concordância Kappa foi de 0,25 nas cristas palmares, 0,06 no leito ungueal e 0,16 na mucosa ocular, valores muito aquém dos considerados satisfatórios para um teste diagnóstico. Este resultado pode ser interpretado como baixa reprodutibilidade do exame de quantificação em cruzes. Spinelli et al. utilizaram outros coeficientes na análise dos dados e apresentaram semelhantes resultados para reprodutibilidade dos exames de palidez palmar e conjuntival ${ }^{13}$. Outro estudo brasileiro, realizado em crianças, também utilizou diferentes coeficientes e obteve reprodutibilidade entre leve e moderada para os sinais clínicos de palidez ${ }^{11}$. Em pesquisa realizada na cidade de Toronto, englobando 302 pacientes acima de 18 anos, houve concordância entre observadores sensivelmente aumentada em relação a estudos anteriores ${ }^{19}$. Os autores atribuem esse aumento à padronização da forma de observação adotada durante os exames e à quantificação de palidez em apenas três níveis.

Pesquisa realizada em crianças, pela Escola Paulista de Medicina, verificou que a probabilidade de serem encontrados um ou mais sinais clínicos de anemia aumenta, conforme a diminuição da hemoglobina no sangue venoso ${ }^{11}$. Outro estudo sugere ainda, que a capacidade de detectar-se anemia grave é maior quando se considera mais de um sinal clínico de anemia ${ }^{17}$.

Novas pesquisas na população adulta, com amostras maiores, heterogeneidade étnica, avaliação de outros sinais clínicos de anemia,

Tabela 1 - Grau de classificação da palidez em cruzes para cristas palmares, de acordo com a média de $\mathrm{Hb}(\mathrm{g} / \mathrm{dL})$, segundo os observadores 1 e 2; HNSC, Tubarão, mar/abr 2007

\begin{tabular}{lcccc}
\hline $\begin{array}{l}\text { Palidez } \\
\text { (grau de classificação) }\end{array}$ & $\begin{array}{c}\text { Observador } 1 \\
\mathrm{Hb}(\mathrm{g} / \mathrm{dL}) / \mathrm{n}\end{array}$ & $\mathrm{dp}$ & $\begin{array}{c}\text { Observador } 2 \\
\mathrm{Hb}(\mathrm{g} / \mathrm{dL}) / \mathrm{n}\end{array}$ & $\mathrm{dp}$ \\
\hline Ausente & $10,48 / 7$ & 3,01 & $11,59 / 10$ & 1,20 \\
$+/ 4$ & $12,48 / 11$ & 1,71 & $12,60 / 7$ & 1,79 \\
$++/ 4$ & $11,54 / 9$ & 1,95 & $9,73 / 9$ & 2,99 \\
$+++/ 4$ & $9,76 / 3$ & 3,71 & $13,07 / 4$ & 2,50 \\
\hline
\end{tabular}

Tabela 2 - Grau de classificação da palidez em cruzes para leito ungueal, de acordo com a média de $\mathrm{Hb}(\mathrm{g} / \mathrm{dL})$, segundo os observadores 1 e 2; HNSC, Tubarão, mar/abr 2007

\begin{tabular}{lcccc}
\hline $\begin{array}{l}\text { Resposta } \\
\text { (grau de classificação) }\end{array}$ & $\begin{array}{c}\text { Observador 1 } \\
\mathrm{Hb} \mathrm{(g/dL)/n}\end{array}$ & $\mathrm{dp}$ & $\begin{array}{c}\text { Observador 2 } \\
\mathrm{Hb}(\mathrm{g} / \mathrm{dL}) / \mathrm{n}\end{array}$ & $\mathrm{dp}$ \\
\hline Ausente & $11,30 / 5$ & 3,51 & $11,43 / 8$ & 2,80 \\
$+/ 4$ & $12,10 / 12$ & 2,25 & $11,53 / 11$ & 1,75 \\
$++/ 4$ & $10,94 / 10$ & 1,54 & $11,18 / 8$ & 3,16 \\
$+++/ 4$ & $10,15 / 3$ & 5,16 & $10,50 / 3$ & 1,55 \\
\hline
\end{tabular}

Tabela 3 - Grau de classificação da palidez em cruzes para mucosa ocular, de acordo com a média de $\mathrm{Hb}(\mathrm{g} / \mathrm{dL})$; HNSC, Tubarão, mar/abr 2007

\begin{tabular}{lcccc}
\hline $\begin{array}{l}\text { Palidez } \\
\text { (grau de classificação) }\end{array}$ & $\begin{array}{c}\text { Observador 1 } \\
\mathrm{Hb}(\mathrm{g} / \mathrm{dL}) / \mathrm{n}\end{array}$ & $\mathrm{dp}$ & $\begin{array}{c}\text { Observador 2 } \\
\mathrm{Hb}(\mathrm{g} / \mathrm{dL}) / \mathrm{n}\end{array}$ & $\mathrm{dp}$ \\
\hline Ausente & $11,24 / 9$ & 2,97 & $12,50 / 7$ & 1,14 \\
$+/ 4$ & $11,97 / 12$ & 1,99 & $11,48 / 10$ & 2,67 \\
$++/ 4$ & $11,65 / 7$ & 2,03 & $11,45 / 7$ & 2,34 \\
$+++/ 4$ & $8,45 / 2$ & 2,75 & $10,05 / 6$ & 3,06 \\
\hline
\end{tabular}


bem como avaliação do grau de experiência dos observadores e treinamento para realização da avaliação, poderiam fornecer dados adicionais referentes à validade externa dos achados aqui descritos.
Os resultados deste estudo demonstraram uma fraca concordância entre observadores, bem como ausência de significância estatística nos diferentes níveis de hemoglobina encontrados para os diferentes graus atribuídos.

\section{Referências}

1. Zago MA, Falcão RP, Pasquini R. Hematologia Fundamentos e Prática. São Paulo: Atheneu: 2004

2. Goldman L, Ausiello D. Tratado de medicina interna. $2^{\mathrm{a}}$ ed. São Paulo: Saunders Elsevier: 2004.

3. Braunwald E, Fauci AS, Kasper DL, Hauser SL, Longo DL, Jameson JL. Medicina interna. 15ª ed. Rio de Janeiro: McGraw Hill: 2002.

4. Haemoglobin colour scale: operational research agenda and study design. World Health Organization 2004. Disponível em URL: www.who.int/entity/medical_ devices/publications/en/Res_Agenda.pdf. Acessado em 12 de agosto de 2006.

5. La anemia como centro de atención: Hacia un enfoque integrado para un controle eficaz de la anemia. Declaración conjunta de la Organización Mundial de la Salud y el Fondo de las Naciones Unidas para la Infancia. 2004. Disponível em URL: http://www.paho.org/Spanish/AD/FCH/NU/OMSO4 Anemia.pdf. Acessado em dez de agosto de 2006.

6. Sari M, Pee S, Martini E, Herman S, Sugiatmi, Bloem MW et al. Estimating the prevalence of anaemia: a comparison of three methods. Bull World Health Organ 2001;79(6):506-11.

7. Neuman NA, Tanaka OA, Szarfarc SC, Guimarães PRV, Victora CG Prevalência de fatores de risco para anemia no sul do Brasil. Rev Saúde Pública 2000;34(1):56-63.

8. Porto CC. Exame clínico: bases para a prática médica. $5^{\mathrm{a}}$ ed. Rio de janeiro: Guanabara Koogan: 2004.

9. Goic A, Chamorro G, Reyes H. Semiologia médica. $2^{\mathrm{a}}$ ed. Santiago: Mediterráneo: 1999.

10. Zucker JR, Perkins BA, Jafari H, Otieno J, Obonyo C, Campbell CC. Clinical signs for the recognition of children with moderate or severe anaemia in western Kenya. Bull World Health Organ 1997;75(Suppl 1):97-102.
11. Sdepanian VL, Silvestrini WS, de Morais MB Limitação diagnóstica do exame físico na identificação de crianças com anemia. Rev Assoc Med Bras. 1996;42(3):169-74

12. Sackett DL, Haynes RB, Guyatt GH, Tugwell P. Clinical epidemiology. A basic science for clinical medicine. 2a ed. Boston: Little Brown and Company: 1991.

13. Spinelli MGN, Souza JMP, Souza SB, Sesoko EH. Confiabilidade e validade da palidez palmar e de conjuntivas como triagem de anemia. Rev Saúde Pública 2003;37(4):404-8.

14. Leal LP, Osório MM. Validação e reprodutibilidade de sinais clínicos no diagnóstico de anemia em crianças. Cad Saúde Pública 2005;21(2):565-72.

15. Kalter HD, Burnham G, Kolstad PR, Hosshain M, Schillinger JA, Khan $\mathrm{NZ}$, et al. Evaluation of clinical signs to diagnose anaemia in Uganda and Bangladesh, in areas with and without malaria. Bull World Health Organ 1997;75(1):103-11.

16. Luby SP, Kazembe PN, Redd SC, Ziba C, Nwanyanwu OC, Hightower AW, et al. Using clinical signs to diagnose anaemia in African children. Bull World Health Organ 1995;73(4):477-82.

17. Zucker JR, Perkins BA, Jafari H, Otieno J, Obonyo C, Campbell CC. Clinical signs for the recognition of children with moderate or severe anaemia in western Kenya. Bull World Health Organ 1997;75(1):97-102.

18. Chalco JP, Huicho L, Alamo C, Carreazo NY, Bada CA. Accuracy of clinical pallor in the diagnosis of anaemia in children: a meta-analysis. BCM Pediatrics 2005;5:46.

19. Sheth TN, Choudhry NK, Bowes M, Detsky AS. The relation of conjuntival pallor to the presence of anemia. J Gen Intern Med 1977;12(2):102-6. 